BRAZILLAN JOURNAL

www.bjournal.com.br
ISSN 0100-879X

Volume 43 (3) 182-267 March 2011

BIOMEDICAL SCIENCES

AND

CLINICAL INVESTIGATION

Braz J Med Biol Res, March 2011, Volume 44(3) 236-239

doi: 10.1590/S0100-879X2011007500007

Gender differences in serum CK-MB mass levels in healthy Brazilian subjects

C.M.C. Strunz, L.M. Araki, A.A.R. Nogueira and A.P. Mansur

The Brazilian Journal of Medical and Biological Research is partially financed by

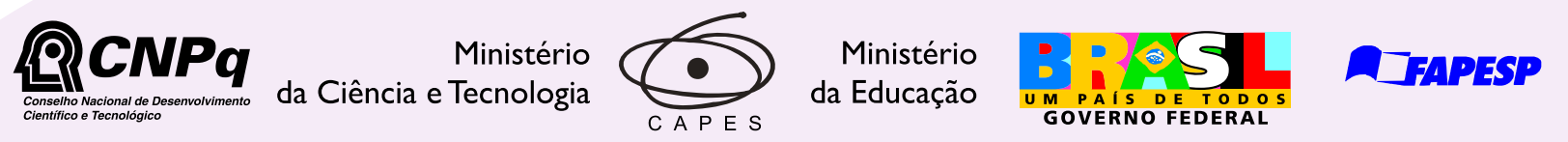

Institutional Sponsors
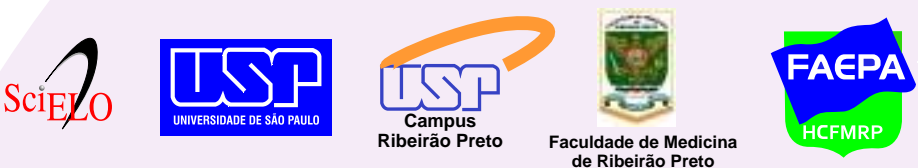


\title{
Gender differences in serum CK-MB mass levels in healthy Brazilian subjects
}

\author{
C.M.C. Strunz, L.M. Araki, A.A.R. Nogueira and A.P. Mansur \\ Laboratório Clínico, Instituto do Coração, Faculdade de Medicina, \\ Universidade de São Paulo, São Paulo, SP, Brasil
}

\begin{abstract}
The creatine kinase-isoenzyme MB (CK-MB) mass assay is one of the laboratory tests used for the diagnosis of myocardial infarction. It is recommended, however, that reference limits should take gender and race into account. In the present study, we analyzed the plasma CK-MB mass and troponin levels of 244 healthy volunteers without a personal history of coronary artery disease and with no chronic diseases, muscular trauma or hypothyroidism, and not taking statins. The tests were performed with commercial kits, CK-MB mass turbo kit and Troponin I turbo kit, using the Immulite 1000 analyzer from Siemens Healthcare Diagnostic. The values were separated according to gender and showed significant differences by the Mann-Whitney test. Mean $( \pm \mathrm{SD})$ CK-MB mass values were $2.55 \pm 1.09$ for women $(\mathrm{N}=121$; age $=41.20 \pm 10.13$ years $)$ and $3.49 \pm 1.41 \mathrm{ng} / \mathrm{mL}$ for $\mathrm{men}$ $(\mathrm{N}=123$; age $=38.16 \pm 11.12$ years $)$. Gender-specific reference values at the 99th percentile level, according to the Medicalc statistical software, were $5.40 \mathrm{ng} / \mathrm{mL}$ for women and $7.13 \mathrm{ng} / \mathrm{mL}$ for men. The influence of race was not considered because of the high miscegenation of the Brazilian population. The CK-MB values obtained were higher than the $5.10 \mathrm{mg} / \mathrm{mL} \mathrm{proposed}$ by the manufacturer of the laboratory kit. Therefore, decision limits should be related to population and gender in order to improve the specificity of this diagnostic tool, avoiding misclassification of patients.
\end{abstract}

Key words: CK-MB mass; Gender; Healthy volunteers

\section{Introduction}

Cardiac troponins are considered to be the gold-standard biomarker for the detection of myocardial injury and, in their absence, the creatine kinase-isoenzyme MB (CK-MB) assay continues to be the other preferred laboratory test. A recent guideline for the redefinition of myocardial infarction has established the 99th percentile of the biomarkers for a reference population as the decision limit for the diagnosis of myocardial necrosis (1). Considering the analytical aspects of the biomarkers, the National Academy of Clinical Biochemistry (NACB) and the International Federation of Clinical Chemistry (IFCC) Committees recommend that each laboratory should establish the reference limit based on a healthy population and taking into account gender and race (2). Few studies establishing gender and ethnic differences for CK-MB mass reference limits are available in the literature (2-4). By analyzing seven CK-MB assays, Apple et al. (3) demonstrated 1.2 to 2.6 higher 99 th percentile values for males vs females and substantial differences based on ethnicity (3). These higher values can be explained by the larger muscle mass found in men compared to women and by between-race differences. Because skeletal muscle is composed of a small amount of CK-MB (1-3\%), it is expected that higher concentrations of this marker will be present in men and blacks (3). As a result of the great miscegenation of the Brazilian population, we are not able to separate groups based on race. On the other hand, gender-specific reference limits should be established and adopted for clinical decisions.

\section{Material and Methods}

Our study cohort comprised 244 healthy volunteers without a personal history of coronary artery disease and with no chronic diseases, muscular trauma or hypothyroidism, and not taking statins (5). They were selected from the employees of the Heart Institute of the Medical School, University of São Paulo. The sample size was based on the guidelines elaborated by the Clinical and Laboratory Standards Institute (CLSI), which recommends a minimum of 120 samples per group of healthy individuals for an adequate

Correspondence: C.M.C. Strunz, Laboratório Clínico, InCor, FM, USP, Av. Dr. Eneas Carvalho Aguiar, 44, $05403-000$ São Paulo, SP, Brasil. Fax: +55-11-3069-5394. E-mail: labcelia@incor.usp.br

Received April 22, 2010. Accepted January 10, 2011. Available online January 21, 2011. Published March 7, 2011. 
statistical establishment of reference limits (6).

The study subjects were divided into two groups according to gender: females $(N=121$; age $=41.20 \pm 10.13$ years $)$ and males $(N=123$; age $=38.16 \pm 11.12$ years $)$. The study participants were instructed not to perform physical exercise for $48 \mathrm{~h}$ before blood collection. Blood was collected by venipuncture and placed in gel separator tubes. After clot formation the tubes were centrifuged and the tests were run immediately after serum separation. The cardiac markers were measured in a chemiluminescent immunometric assay (turbo CK-MB mass and turbo Troponin I) using the Immulite 1000 analyzer (Siemens Healthcare Diagnostics Inc., UK). The procedure consists of the binding of the molecule being measured (CK-MB or Troponin I) to an antibody-coated bead, where it is captured and held. Then, a second antibody, a murine monoclonal antibody labeled with alkaline phosphatase, binds to the captured substance being held on the bead. A chemiluminescent substrate is added to the test unit and the alkaline phosphatase label catalyzes the substrate and photons of light are released. The photon light output is measured by a Photomultiplier tube and converted to count per second (CPS).

Troponin I was run together with CK-MB mass to increase the chance of detecting any myocardial damage in the study group. The within-laboratory precision, within-run and between-run precision, expressed as coefficient of variation (CV), were determined using commercial control samples (level 1 from Siemens Healthcare Diagnostics Inc.) processed in duplicate over 30 days for a total of 60 replicates. The D'Agostino-Pearson test was used to establish normal or nonparametric value distribution. The nonparametric percentile method was performed according to the standard protocol of CLSI C-28-A3 (6). Suspected outliers were eliminated using the Tukey method, which defines outliers as three interquartile ranges below the 25th percentile or above the 75th percentile. The Mann-Whitney test was performed to determine whether gender-related values were different. The 99th percentile reference interval was determined using the Medicalc statistical software, version 9.6.4.0, Belgium. $\mathrm{P} \leq 0.05$ was considered to be statistically significant.

\section{Results}

Total and gender-specific values for CK-MB mass (ng/ $\mathrm{mL}$ ) are reported in Table 1. The results for both genders as a whole (total) and for the female group followed a nonparametric distribution, whereas the values for the male group followed a normal distribution. The 99th percentile for total CK-MB was $6.63 \mathrm{ng} / \mathrm{mL}$, higher than the value suggested by the manufacturer of our assay $(5.10 \mathrm{ng} / \mathrm{mL})$. Concerning the gender-related median, a significant difference was found in the values for the male group, which were higher than the values for the female group (3.49 vs $2.55 \mathrm{ng} / \mathrm{mL}$; $P<0.0001$; Figure 1). Likewise, the 99th percentile was 1.3-fold higher in men compared to women (7.13 vs 5.40 $\mathrm{ng} / \mathrm{mL}$; Table 1).

Recent guidelines recommend that the cardiac marker assays should have a total imprecision of $10 \%$ at the medical decision limit (99th percentile) $(1,2)$. For CK-MB mass, the assay had a CV of $5.6 \%$ at a concentration of $6.48 \mathrm{ng} / \mathrm{mL}$, a value close to the medical decision limits established in our study. For troponin I, the CV obtained was $8.59 \%$ at a concentration of $0.84 \mathrm{ng} / \mathrm{mL}$. Based on our results, we adopted a reference limit for troponin I of $0.6 \mathrm{ng} / \mathrm{mL}$, as suggested by another study in the literature (3), as the concentration that will reach the analytical goal proposed by others $(1,2)$. Almost all the troponin I obtained

Table 1. CK-MB mass concentrations by gender.

\begin{tabular}{lccc}
\hline & Total & Males & Females \\
\hline Sample size & 244 & 123 & 121 \\
Concentration range $(\mathrm{ng} / \mathrm{mL})$ & $0.72-6.64$ & $0.73-7.19$ & $0.72-5.40$ \\
Mean and standard deviation $(\mathrm{ng} / \mathrm{mL})$ & $3.03 \pm 1.34$ & $3.49 \pm 1.41$ & $2.55 \pm 1.09$ \\
95\%Cl of the mean $(\mathrm{ng} / \mathrm{mL})$ & $2.86-3.20$ & $3.24-3.75$ & $2.35-2.74$ \\
Median $(\mathrm{ng} / \mathrm{mL})$ & 2.95 & $3.34^{*}$ & 2.33 \\
95\%Cl of the median $(\mathrm{ng} / \mathrm{mL})$ & $2.64-3.15$ & $3.12-3.63$ & $2.09-2.62$ \\
99th percentile $(\mathrm{ng} / \mathrm{mL})$ & 6.63 & 7.13 & 5.40 \\
$90 \% \mathrm{Cl}$ of the 99 th percentile $(\mathrm{ng} / \mathrm{mL})$ & NA & $6.77-7.50$ & NA \\
\hline
\end{tabular}

CK-MB mass = creatine kinase-isoenzyme MB mass; NA = not applicable. ${ }^{*} \mathrm{P}<$ 0.0001 for males vs females (Mann-Whitney test).

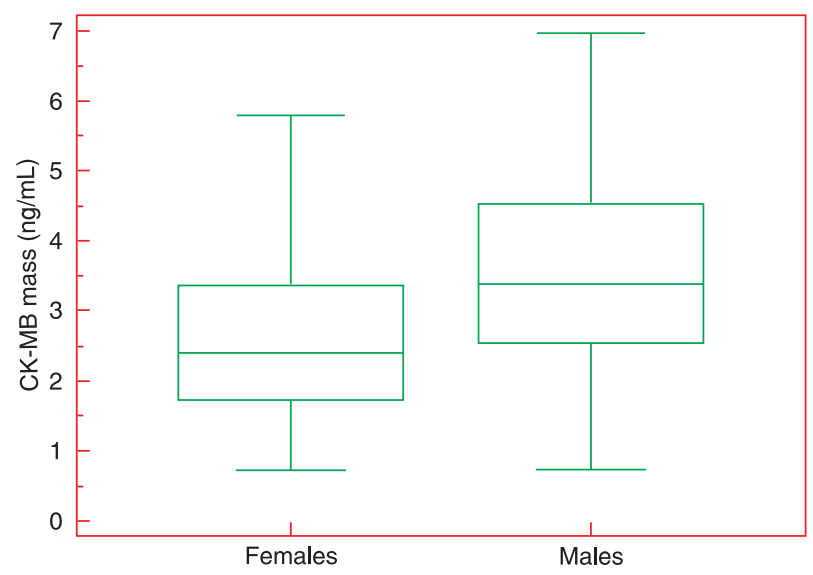

Figure 1. Comparison of female and male CK-MB concentrations. Box-and-whisker representation of the results. CK-MB mass $=$ creatine kinase-isoenzyme MB mass. CK-MB values for males $(N=123)$ were significantly different from those for females $(\mathrm{N}=121 ; \mathrm{P}<0.0001$, Mann-Whitney test). 
from volunteers resulted in values $<0.2 \mathrm{ng} / \mathrm{mL}$, indicating the absence of myocardial necrosis.

\section{Discussion}

For the diagnosis of myocardial necrosis, the NACB recommends the use of $\mathrm{CK}-\mathrm{MB}$ measurements in two consecutive samples exceeding the 99th percentile of values for a gender-specific reference control group (7). Unfortunately, few manufacturers report medical decision limits for the CK-MB mass at the 99th percentile level and based on gender specificity. Reference limits for CK-MB mass in women and men were different in our population and similar literature results (Table 1 ) $(3,4)$. This is probably the consequence of a greater body muscle mass in men and indicates the importance of different decision-limit values based on gender in clinical practice. The 99th percentile claimed by the in vitro diagnostic company in their package insert used in our laboratory is $5.10 \mathrm{ng} / \mathrm{mL}$, without any information about a gender-specific reference range. In the present study, two decision limits were established for the values at the 99th percentile level: $5.40 \mathrm{ng} / \mathrm{mL}$ for women and $7.13 \mathrm{ng} / \mathrm{mL}$ for men. The gender-related values and the total population cut-off were higher than those suggested by the manufacturer. The disagreement in results could be explained by differences in race composition between the populations. Four CK-MB assays tested by Apple et al. (3) showed higher (1.2- to 2.7-fold; P < 0.02) mean concentrations for blacks compared to Caucasians. Henny and Hyltoft (8) suggested that decision limits should be adapted to the population because of the differences detected in analyses in some of them. Thus, regional and racial differences may influence the level of many biomarkers. The authors mentioned the impact of ethnicity in developing countries and the difficulty to compare the data produced by these countries to the results reported in developed ones. Unfortunately, the manufacture makes no mention of the race of its reference group. Because of the high miscegenation of our population, a race influence on CK-MB levels is unlikely.

These new reference ranges of CK-MB values were applied retrospectively to 216 consecutive samples of patients with symptoms of acute coronary syndromes (ACS), who were included in a research protocol performed in our hospital (data not shown). A total of 112 study samples were from the male group: 26 from patients with ACS and 86 from patients with other cardiac causes. In the latter group, $25.6 \%$ would have been misclassified if had used the manufacturer reference range instead of our own. No misclassification was found in the ACS group. Concerning the female group ( $\mathrm{N}=104)$, because the cut-off was close to that of the manufacturer, only $5(5.6 \%)$ patients would have been misclassified.

One question raised is why not definitely replace the CK-MB test with the troponin test, which is considered to be the gold standard of cardiac markers for myocardial infarction (1). A College of American Pathologists survey study of the turnaround time of cardiac markers performed at 159 hospitals, mainly in North America, in 2004, showed that CK-MB was still used by $92.7 \%$ of the participating laboratories (9). In a survey conducted during the 2008 audio conference of the American Association for Clinical Chemistry, only $22.2 \%$ of the participating laboratories used only troponin as a cardiac biomarker, whereas about $63.3 \%$ performed both troponin and CK-MB mass tests (10).

We must also consider that, in Brazil, Public Health Insurance does not cover the troponin test, but only the CK-MB test. Finally, with the advent of high-sensitivity tests, very low amounts of circulating troponin are detectable even in a reference population. In a study conducted by Omland et al. (11), troponin levels were associated with the incidence of cardiovascular death and heart failure in a population with stable coronary artery disease, but not independently associated with the incidence of myocardial infarction. Thus, the authors pointed out that, as the troponin tests become more sensitive, the low-level elevations of troponin will be less specific for acute myocardial injury. Taking into account these considerations, we believe that the association of the troponin assay with another biochemical marker like CK-MB mass will increase the specificity of the 2 markers for the detection of myocardial injury.

Therefore, we suggest the cut-off values for CK-MB of $7.1 \mathrm{ng} / \mathrm{mL}$ for men and $5.4 \mathrm{ng} / \mathrm{mL}$ for women in our population. The adoption of decision limits for CK-MB based on gender will improve the specificity of this diagnostic tool, avoiding misclassification of patients, which overwhelms emergency rooms, with social implications for health insurance and employment.

\section{References}

1. Thygesen K, Alpert JS, White HD. Universal definition of myocardial infarction. Eur Heart J 2007; 28: 2525-2538.

2. Apple FS, Jesse RL, Newby LK, Wu AH, Christenson RH. National Academy of Clinical Biochemistry and IFCC Committee for Standardization of Markers of Cardiac Damage Laboratory Medicine Practice Guidelines: Analytical issues for biochemical markers of acute coronary syndromes. Cir- culation 2007; 115: e352-e355.

3. Apple FS, Quist HE, Doyle PJ, Otto AP, Murakami MM. Plasma 99th percentile reference limits for cardiac troponin and creatine kinase MB mass for use with European Society of Cardiology/American College of Cardiology consensus recommendations. Clin Chem 2003; 49: 1331-1336.

4. Zaninotto M, Mion MM, Novello E, Delprete E, Moretti M, 
Plebani M. Creatine-kinase MB mass: age and sex-associated reference limits in two different platforms that use the same method. Clin Chim Acta 2009; 401: 162-164.

5. Pierce GF, Jaffe AS. Increased creatine kinase MB in the absence of acute myocardial infarction. Clin Chem 1986; 32: 2044-2051.

6. Anonymous. Clinical and Laboratory Standards Institute (CLSI) C-28A3. Defining, establishing, and verifying reference intervals in the clinical laboratory; approved guideline. 3rd edn. Wayne: CLSI; 2008.

7. Morrow DA, Cannon CP, Jesse RL, Newby LK, Ravkilde J, Storrow AB, et al. National Academy of Clinical Biochemistry Laboratory Medicine Practice Guidelines: clinical characteristics and utilization of biochemical markers in acute coronary syndromes. Clin Chem 2007; 53: 552-574.
8. Henny J, Hyltoft PP. Reference values: from philosophy to a tool for laboratory medicine. Clin Chem Lab Med 2004; 42: 686-691.

9. Novis DA, Jones BA, Dale JC, Walsh MK. Biochemical markers of myocardial injury test turnaround time: a College of American Pathologists Q-Probes study of 7020 troponin and 4368 creatine kinase-MB determinations in 159 institutions. Arch Pathol Lab Med 2004; 128: 158-164.

10. http://www.aacc.org/publications/cln/2009/april/Pages/ CovStory1April09.aspx.

11. Omland T, de Lemos JA, Sabatine MS, Christophi CA, Rice MM, Jablonski KA, et al. A sensitive cardiac troponin T assay in stable coronary artery disease. N Engl J Med 2009; 361: 2538-2547. 